\title{
Use of LTE for the Interoperability between Different Generations of Wireless Communication
}

\author{
Dilshad Mahjabeen $^{1 *}$, Anis Ahmed ${ }^{2}$, Shahida Rafique ${ }^{2}$ \\ ${ }^{1}$ Department of Electrical and Electronic Engineering, Stamford University, Dhaka, Bangladesh \\ ${ }^{2}$ Applied Physics, Electronics and Communication Engineering, University of Dhaka, Dhaka, Bangladesh \\ E-mail: $\left\{{ }^{*}\right.$ dil_shadman,anis_apedu\}@yahoo.com \\ Received May 10, 2011; revised June 19, 2011; accepted June 30, 2011
}

\begin{abstract}
This paper looks potential approaches to interoperability as well as inter-technology mobility using Long Term Evolution (LTE) between different generations of wireless communication. Focus is given on how these approaches can be used in interoperable service deployment, access technology integration, service continuity and smooth migration to LTE by maximizing the use of legacy networks coverage. One of the most important interoperability criterions is the handover latency. Using NCTUNS6 simulator, it is shown that LTE (4G) provides less handover latency in comparison to that of other generations of wireless communication system for homogeneous environment.
\end{abstract}

Keywords: Interoperability, LTE, Inter-Technology Mobility, Smooth Handover

\section{Introduction}

With the fast-changing mobile landscape and convergence in all aspects of telecommunications, interoperability is important for any technology to succeed. Operators and consumers both benefit from interoperability in terms of cost effectiveness, enhanced features, location independence and ease of use [1,2]. 1G, 2G and 2.5G are very far from this facility. Although 3G (e.g. EVDO, WCAMA developed in 2005) allows simultaneous use of speech and data services with higher data rates but it does not provide mobility and service portability since it is based on primarily a wide-area concept. This great opportunity will be fulfilled by $4 \mathrm{G}$ wireless communications using Long Term Evolution (LTE) as this is based on digital packet networks. This utilizes IP in its fullest form with converged voice and data capability [3].

Developed in 2007, LTE is a step toward the 4G of radio technology. It provides mobility and service portability not only within $4 \mathrm{G}$ network but also between networks including 3.5G (WiMAX), 3G (UMTS) and 2G (GSM and CDMA2000). The LTE aims to provide an all IP backbone with reduction in cost per bit, better service provisioning, flexibility in use of new and existing frequency bands, simple network architecture with open interfaces and lower power consumption [4,5]. The characteristics of LTE are presented in Section 2. In Section
3 , the way of providing interoperability between different generations of wireless communication and intertechnology mobility using LTE are described. The results of simulation are given in Section 4.

\section{Characteristics of LTE}

Subscribers need the same Internet experience that they have at home, anything anywhere. Existing wireless access technology such as HSPA and EVDO go part of the way in meeting this need. But spectral efficiency, celledge capacity and high latency prevent them from providing the bandwidth capacity and QoS to enable a true broadband service. Moreover, the data usages in current network are currently increasing. In response to these dynamics, LTE is introduced. LTE, also known as "Evolved UTRA and UTRAN", is a step toward the 4G of radio technology designed to increase the capacity and speed of mobile telephone (LTE Air information network). LTE innovation goes on iron discussion for its greater bandwidth flexibility, modulation and access schemes $[4,6]$. All the special and differentiating characteristics of LTE are summarized in Table 1.

LTE exploits inter-technology mobility to support a variety of access technologies including 3GPP legacy technologies as well as EVDO, WiFi and WiMAX. This is one of the prime steps for ensuring interoperability. 
Table 1. Features of LTE.

\begin{tabular}{ll}
\hline Features & \\
\hline OFDMA for DL & To achieve high peak data rates (326 Mbps-uplink and 86.4Mbps-downlink) \\
SC-OFDMA for UL & To achieve high Peak to Average Ratio (PAR) of 2 to 6 dB. \\
Scalable & Because of scalable bandwidth up to 20MHz (covering 1.4, 3, 5, 10, 15 and 20MHz) easy migration is possible. \\
Bandwidth & Larger channel bandwidths \\
Spectral efficiency & Increased Spectral Efficiency \\
Modulation & 64QAM \\
Duplexing & Both TDD and FDD profiles \\
Access Network & Flexible \\
UE & Simplified Rx design in UE for high-speed data \\
Antenna Type & MIMO UL \& DL , Collaborative MIMO \\
Interference & Overcome Multi-path Interference \\
Link Capacity & Increased Link Capacity \\
Flat IP & To support voice in the packet domain \\
Latency & Low latency \\
Interoperability & Flexible \\
Mobility & Inter-technology Mobility, Mobile IP based IP Mobility \\
Transport & Backhaul based on IP / MPLS transport \\
& Ensuring Applications at lower cost like \\
Applications & $*$ Peer to peer application requiring high throughput \\
& $*$ Online gaming \\
\hline & $*$ Fits with IMS, VoIP, SIP \\
\hline
\end{tabular}

Inter-technology mobility is the ability to support movement of a device between differing radio access networks. Inter-technology mobility provides the ability to tie together disparate radio access networks, based on different access technologies, into a single integrated bandwidth delivery vehicle. In general, the LTE standards define two types of inter-technology mobility:

1) Inter-RAT (Radio Access Technology) mobility: Mobility between LTE and earlier 3GPP technologies;

2) Inter-Technology mobility: Mobility between LTE and non-3GPP technologies [7].

Inter-technology mobility is the key feature of smooth handover, which ultimately results in interoperability. Seamless handover is one of the mandatory issues of interoperability. In LTE there are three types of handovers:

1) Intra-LTE: Handover happens within the current LTE nodes (intra-MME and Intra-SGW);

2) Inter-LTE: Handover happens toward the other LTE nodes (inter-MME and Inter-SGW);

3) Inter-RAT: Handover between different radio technology networks, for example GSM/UMTS and UMTS [8].

The interoperability offers network providers and users a possibility to choose between alternative wireless access networks [9]. In wireless communication interop- erability, study explores and analyses network performances in terms of packet loss, round-trip time (RTT) and handoff latency (HOL) [10]. Handover latency is the time difference between the moment the MN detects the coverage of another wireless technology and the receive of an acknowledgement from the CN. The access point's (AP's) interoperability with other APs relies on intra, inter handoff process, and frame forwarding. Fast handoff i.e. low handoff latency without Mobile IP eliminates frame losses during handoff [11].

\section{Interoperability Technique by LTE}

Interoperability can offer network providers with a possibility to switch between alternative wireless access networks. This feature provides capability to automatically roam onto a visited network, have access, and share appropriate information or services as authorized. To provide interoperability between different generations of wireless communication following factors are pre-eminent [12]:

1) Affordability and availability of Customer Premises Equipment (CPE);

2) Reusability/upgradeability of base stations;

3) Acquisition of additional spectrum;

4) Frequency Planning; 
5) Capacity Planning;

6) Flexibility of Access Service Network (ASN) gateways;

7) Supporting ecosystem of devices.

LTE architecture has several defined interfaces (SIMME, SI-U, S3-SS12, SGi etc) as shown in Figure 1 for interoperating with the network. These interfaces will allow users to roam into networks via these interfaces. LTE provides interoperability that allows users to leave their home and authenticate automatically into a visited network. This feature also provides access to public internet access, best effort data and VPN access to their home network. LTE provides interoperability between different network by seamless and lose less handover. As for example, $700 \mathrm{MHz}$ LTE networks ensure the internetworking connectivity [2]. Figure 1 shows the flexible interoperability by LTE. In this particular technique, two specific nodes such as Serving Gateway (SGW) and Mobility Management Entity (MME) are used. Existing GSM, W-CDMA, HSPA can be integrated into LTE network through some standardized interfaces (SI-MME, SI-U, S3-SS12, SGi etc) between Serving GPRS Support Node (SGSN) in case of 3GPP and between PSDN in case of 3GPP2.

Presently vendors are providing $2 \mathrm{G}$ and $3 \mathrm{G}$ built in interoperability within SGSN and PDSN to LTE core network. All IP technology like WiMAX can interface in similar fashion to LTE core network. The Packet Data Network Gateway (PGW) is responsible for IP address allocation for user equipment (UE) as well as QoS enforcement. When UE is attached to a network, it is assigned an IP address by the PGW and the fourth bearer is established. The preface of fourth bearer is to provide always on connectivity. The PGW serves as a mobile anchor for interworking with non-3GPP technology such

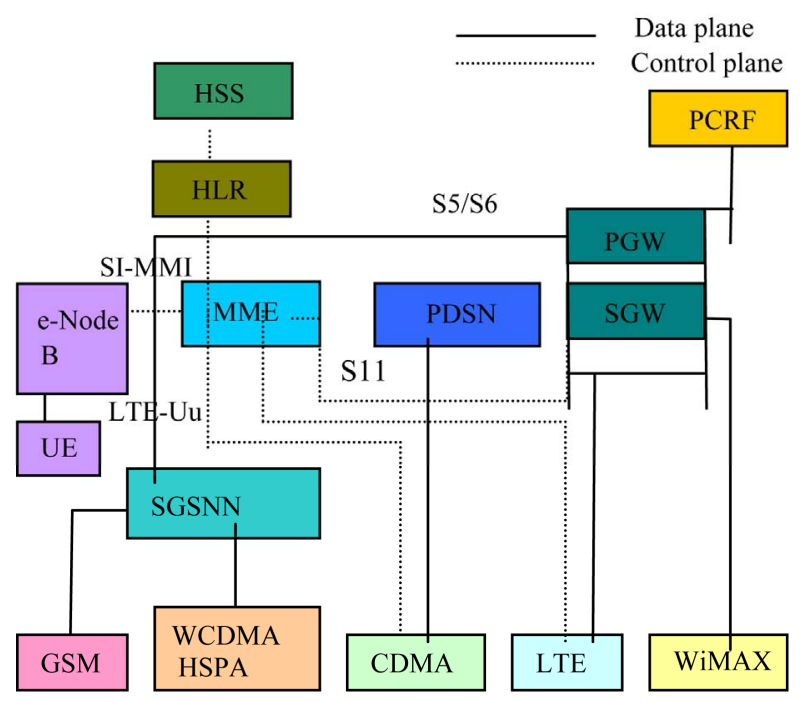

Figure 1. Flexible interoperability by LTE. as cdma-2000 and WiMAX. Lastly, HSS, HLR contains usage subscriber information such as QoS at any access restrictively for roaming. Both the facilities can be used to solve the problems in service deployment, access technology integration and smooth migration [13].

In 2G and 3G wireless communication, Radio Network Controllers (RNC) serves as the main functional unit of radio access network. Serving GPRS support node (SGSN) and gateway GPRS support node (GGSN) network elements provide the packet data services in the networks. 3GPP Release 7 is just the previous version of LTE. In direct tunnel solution, user plane will bypass SGSN as shown in Figure 2. This will increase the flexibility in network topology and allows the SGSN node to be optimized for control plane. This is the first step towards system architecture evolution (SAE) which is the base for LTE. Data in I-HSPA bypasses the radio network controller (RNC) and the SGSN, and offloads data directly to the Internet via GGSN. This reduces the capacity bottlenecks in critical network elements like RNC and SGSN. However 3GPP Release8-LTE defines an all-IP network as a base for the LTE/SAE. The LTE/SAE does not have separate packet switched data traffic and circuit switched voice network. Both data and user plane communicates over the same network, which is called Evolved Packet System (EPS) network [14]. Like 3GPP Release 7, LTE embeds radio controller functionalities into eNodeB which allows tight interaction between the protocol layers of AN. This distributed control eliminates the need for a processing intensive radio controller, which in turn reduces the cost and avoids a "single point of failure".

In addition, due to absence of the radio controller improve the efficiency of the network by reducing the latency. There is no soft handover in LTE, which eliminates the need for a centralized data combining function. Practically, in this arrangement for 2G/3G/4G interworking features it platform i.e. easy, efficient and flexible BTS and multi-radio sites are used for all technologies [13]. With the introduction of LTE, the limitation of access network interconnection to technologies covered by the same standards is changing. LTE Standards accommodate the use of Mobile Internet Protocol MIP to support inter-technology mobility between LTE an WiFi and also between EVDO and WiMAX [4].

Interoperability of LTE with other generations are discussed in the following sections:

\subsection{Interoperability between 3.5 G (WiMAX) and LTE}

3.5G-WiMAX and LTE will complement each other. WiMAX is a next-generation technology that will facilitate the cellular operators' transition to all-IP networks. 

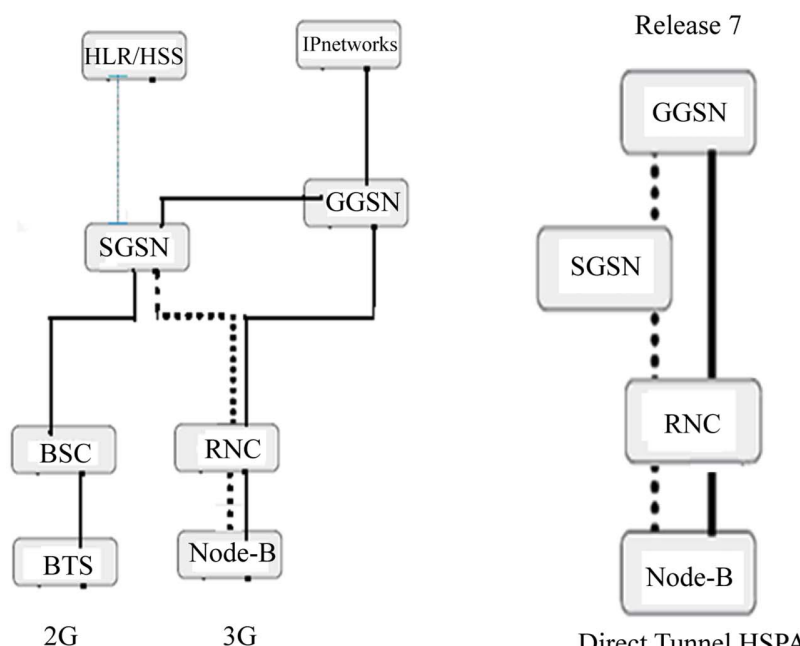

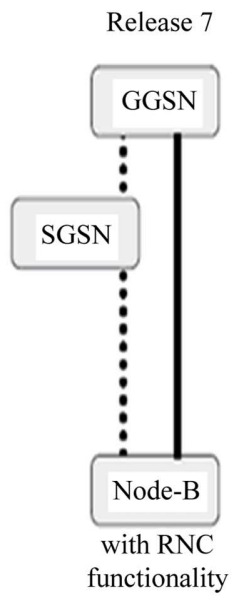

1-HSPA

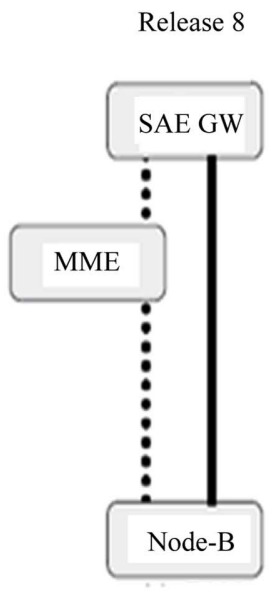

LTE

Figure 2. Comparison between network architecture of different generations of wireless communication and LTE.

The IP core network at the basis of WiMAX will simplify interworking with other IP technologies like LTE and System Architecture Evolution (SAE). WiMAX fully supports IMS and its 3GPP2 LTE counterpart. Support for IMS will facilitate interworking with flexible layered architecture and remove existing redundancies in the core network $[14,15]$. Integration of WiMAX with LTE assures a unified network architecture that facilitates interworking, roaming and infrastructure sharing with current and emerging cellular and wired technologies $[13,16]$.

The prime and main step of interoperability is easy and smooth migration from one technology to another of different generations of wireless communication. For a practical but smooth and easy migration from WiMAX 16e to LTE technology, a practical but smooth and easy migration through a combination of software upgrades and straightforward blade server (i.e. base band card) change out should be considered. Network transition from WiMAX to LTE is shown in Figure 3.

The BS of LTE can be found by using a new software

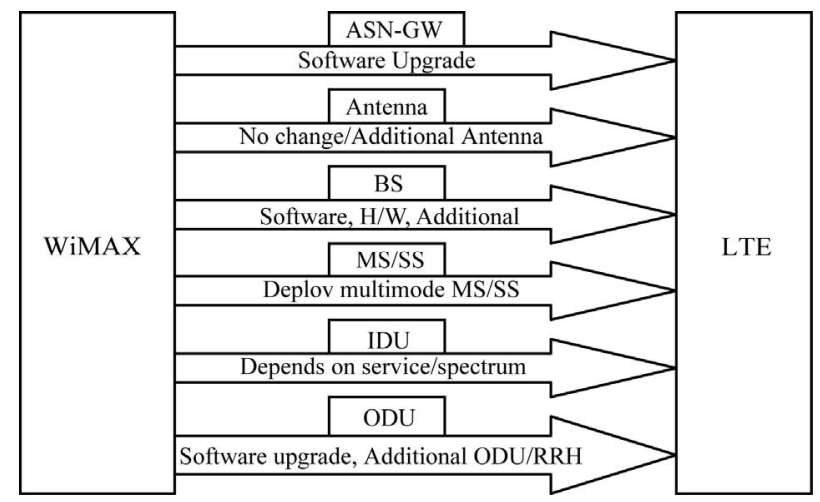

Figure 3. Migration from WiMAX 16e LTE technology. and hardware with an existing BS of WiMAX [17]. Similarly, other changes are shown in the middle column in Figure 3. As TDD ratio of WiMAX and LTE is not same, so frame synchronization is required by sacrificing some symbols of WiMAX [2].

\subsection{Interoperability between 3G (UMTS) and LTE}

In the LTE-to-UMTS Inter RAT handover, the source e-NodeB connects to the MME and SGW while the target RNC connects to the SGSN and SGW; both the source and target SGWs connect to the same PGW. This procedure is divided into two parts for clarity, Preparation and Execution. In the Preparation phase, resources are reserved in the target network. In the Execution phase, the UE is handed over to the target network from the source network [8].

\subsection{Interoperability between 2G (GSM) and LTE}

LTE platform provides a view of interoperability for the carriers across their SIP and SS7 signaling domains. In this approach, information is sent from a $2 \mathrm{G}$ device, controlled within the SS7 environment and then send a message and have it read by a user within an LTE/IMS environment. LTE uses IP Short Message Gateway (IP-SMGW) which supports SMS and MMS in all-IP networks, and that allows operators to interwork with $2 \mathrm{G}$ networks [8].

\section{Simulation Results}

In this section results of simulation which was done with 
the help of nctuns6 simulator [18] are presented. In wireless communication interoperability, study explores and analyses network performances in terms of packet loss, round-trip time (RTT) and handoff latency [10]. In this paper, parameter of interest is the experienced handover latency (HOL). In homogeneous environments, the handover behavior is analyzed. The analyzed simulation scenario investigates handover effects between different generations of wireless communications such as GPRS (for 2G), IEEE 802.11b (WiFi for 3G) and IEEE 802.16e, j (WiMAX for $4 \mathrm{G}$ ). The simulation follows the steps in Figure 4.

It consists of a Corresponding Node (CN) generating traffic towards a Mobile Node (MN), intermediate routers, IEEE 802.11(b) Access Point (AP), IEEE 802.16(e) and IEEE802.16 (j) Base Station (BS) and a GPRS node placed in a simulated area of $2000 \mathrm{~m} \times 2000 \mathrm{~m}$. The whole simulation area has GPRS coverage, whereas the IEEE 802.16 and the IEEE 802.11 technologies cover a circular area with a radius of $500 \mathrm{~m}$ and $40 \mathrm{~m}$ inside, respectively. The MN moves freely throughout the simulation area performing various vertical handover. In edit section, the following parameters are considered (Table 2).

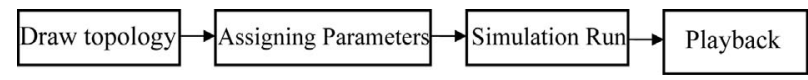

Figure 4. Steps of simulation process.

Table 2. Module Parameter Setting.

\begin{tabular}{lc}
\hline Parameter & Value \\
\hline Fading Variance & 10 \\
Average Building Height (m) & 10 \\
Average Building distance (m) & 80 \\
Street width (m) & 30 \\
Path loss exponent & 2 \\
Shadowing Standard deviation & 4 \\
Close in reference distance (m) & 1 \\
System loss & 1 \\
BS Antenna Height (m) & 30 \\
Ricean factor (db) & 10 \\
Propagation channel model & Theoretical channel model \\
Empirical channel model & COST HATA231 \\
Frequency (MHz) & 2300 \\
T Power (dBm) & 35 \\
Receiver Sensitivity & -96 \\
Transmission Mode & Full duplex \\
MN antenna height (m) & 1.5 \\
Traffic bit rates & TCP \\
& \\
&
\end{tabular}

The MN's speed also varies depending upon the surrounding environmental conditions. As for example in this work, maximum speed of 802.11(b), 802.16(e), 802.16(j) reaches 20, 28 and $36 \mathrm{~m} / \mathrm{s}$ respectively. MN,s speed is also related to handover latency. Based upon the parameters as stated in Table 2 the simulation is performed and the obtained results (Figures 5-8) are compared with the HOL of LTE.

In Figure 5 the handover latency of GPRS is found from $6 \mathrm{~s}$ to $9 \mathrm{~s}$ in the time axis. Thus from Figures 5-8 the total handover latency of GPRS, IEEE 802.11b, IEEE 802.16e and IEEE 802.16j are found to be $4 \mathrm{~s}, 4 \mathrm{~s}, 3 \mathrm{~s}$ and $1 \mathrm{~s}$, respectively. This result shows that the handover latency of GPRS (2G) and IEEE 802.11b (3G) are the highest while that of IEEE $802.16 \mathrm{j}$ (4G) is the lowest. So

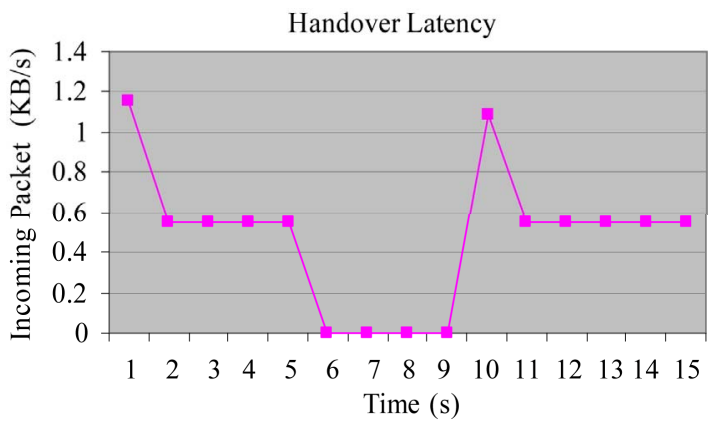

Figure 5. HOL in GPRS system.

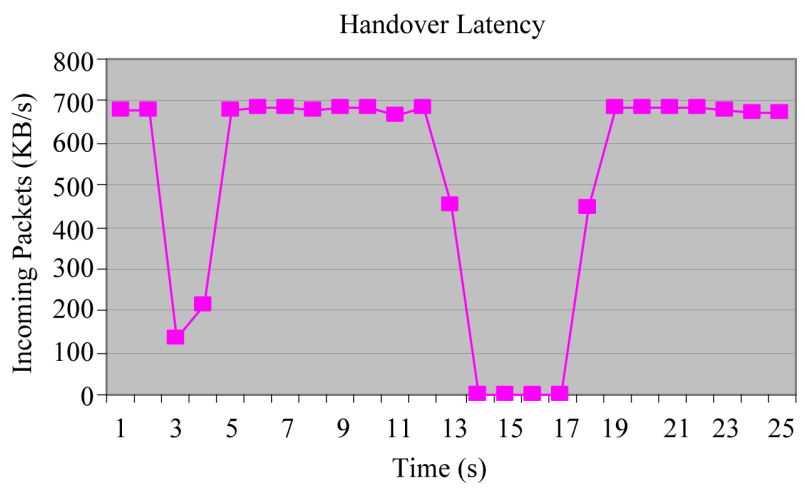

Figure 6. HOL in 802.11b.

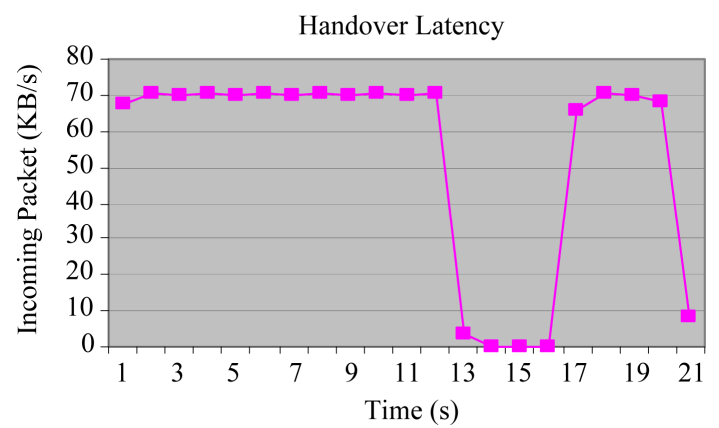

Figure 7. HOL in mobile WiMAX 802.16e. 


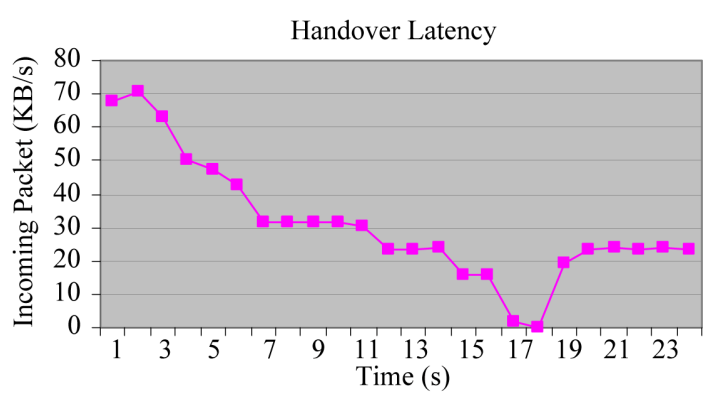

Figure 8. HOL in Mobile WiMAX802.16j.

HOL is gradually decreasing from $2 \mathrm{G}$ to $3 \mathrm{G}$ to $4 \mathrm{G}$ and hence supporting the analysis that $4 \mathrm{G}$ wireless communication system is more interoperable. The experimental maximum range of handover latency of LTE is generally 92 up to 96.5 ms even with an error rate of $15 \%$ in cells within radius $1 \mathrm{~km}$ [19]. So as a part of 4G, LTE also provides less handover latency than GPRS (2G) and IEEE 802.11b (3G) and ensures more interoperability as well as more flexibility with other generations of wireless communication.

\section{Conclusions}

LTE is considered as the basis of next generation mobile Internet. LTE Standards accommodate the use of Mobile Internet Protocol (MIP) to support inter-technology mobility between LTE and other generations. In our work, different ways of providing interoperability by LTE and use of interfaces for flexible interoperability are discussed. In addition, we focus on the variation of network archicture of different generations including LTE, which leads to the interoperability. Moreover, method of migration from $3 G$ to $4 G$ is highlighted. Result of hand- over latency for homogeneous network has shown that handover latency in LTE is less than GPRS (2G) and IEEE 802.11b (3G). This result proves that LTE (4G) provides better interoperability performance than other generations. In our future work, we will determine handover lancy for heterogeneous environments.

\section{References}

[1] “Long-Term Evolution (LTE): The Vision Beyond 3G," 4gwirelessevolution.tmcnet.com/.../Nortel\%20LTE\%20T he\%20vision\%20beyond \%203G.pdf

[2] “Technical Papers on LTE-Advanced and IEEE 802.16m,” 2011. www.beyond4g.org

[3] D. I. Axiotis, F. I. Lazarakis and C. Vlahodimitro, "4G System Simulation Parameters for Evaluating the Interoperability of MTMR in UMTS and HIPERLAN/2," Proceedings of the 54th IEEE Semiannual Vehicular Technological Conference, Jeju, Vol. 4, Spring 2003, pp. 2745-2749.
[4] NPSTC, "Broadband Task Force Governance Group Roaming White Paper,” 2011.

http://www.napco.org/Resources/700_MHz_BBTF_Final _Report_9-4-2009.pdf

[5] LTE Advanced LteWorld, 2011. lteworld.org/category/tags/lte-advanced

[6] D. Astely, E. Dahlman, A. Furuskar, Y. Jading, M. Lindstrom and S. Parkvall, "LTE: The Evolution of Mobile Broadband," IEEE in Communication Magazine, Vol. 47, No. 4, 2009, pp. 44-51. doi:10.1109/MCOM.2009.4907406

[7] "LTE Inter technology Mobility: Enabling Mobility between LTE and Other Access Technologies,” 2011. motorola.com/.../Document/Static Files/ 6834_MotDoc

[8] V. S. Rao and R. Gajula, "Interoperability in LTE,” 2011. www.ccpu.com/articles/2010/interoperability-lte

[9] “MIH Performane,” 2011. www.scribd.com/doc/53408917/MIH-Performance

[10] L. Grymek, S. Singh and K. Pattipati, "Embeded Worldwide Interoperability for Microwave Access Based Router Vehicular Telematics Computing,” 2011. ieeexplore.ieee.org/iel5/4105970/5454243/05454255

[11] C.-T. Chou and K. G. Shin, “An Enhanced Inter-Access Point Protocol for Uniform Intra and Intersubnet Handoffs," IEEE Transaction on Mobile Computing, Vol. 4, No. 4, July-August 2005, pp. 321-334. doi:10.1109/TMC.2005.49

[12] "WiMAX 16e: Evolutionary Choices between16M and TD-LTE,” 2011. www.aviatnetworks.com

[13] S. Sesia, I. Toufik and M. Baker, "LTE-The UMTS Long Term Evolution: From Theory to Practice,” John Wiley \& Sons Ltd, Hoboken, 2009, p. 24. doi:10.1002/9780470742891

[14] G. Fritze, “The Core Network for LTE. Retrieved 3 2,” 2009.

http://www.nt.tuwien.ac.at/fileadmin/users/wkarner/2008 -04-10_SAE.pdf

[15] Dillshad Mahjabeen, A. H. M Sayem, A. Ahmed and S. Rafique, "Interoperability of Wireless Communication with $4 \mathrm{G}$ based on Layer Modification,” International Journal of Communications, Network and System Sciences, Vol. 3, No. 5, 2010, pp. 474-478.

[16] K. S. Keshava Murty, "Next Generation Wireless Access Gateway Analysis of Combining WiMAX and LTE Gateway Functions," Proceeedings of the 2nd International Conference on Internet Multimedia Services and Applications, Bangalore, 2008, pp. 1-6.

[17] S. S. Wagle, M. Ade and M. G. Ulla, "Network Transition from WiMAX to LTE,” Journal of Computing, Vol. 3, No. 1, January 2011, ISSN 2151-9617. https://sites.google.com/site/journal computing/ www.journalcomputing.org70

[18] “NCTUns Network Simulator and Emulator,” 2011. nsl.csie.nctu.edu.tw/nctuns.html

[19] K. Dimou, M. Wang, Y. Yang, M. Kazmi, A. Larmo, J. Pettersson, W. Muller and Y. Timner "Handover within 3GPP LTE: Design Principles and Performance,” 2011. www.ericsson.com/res/thecompany/docs/.../VTC09F_HO _LTE.pdf 